\title{
Osteopatía hipertrófica secundaria a metástasis pulmonar de carcinoma mamario
}

Ricardo Andrés Correa-Salgado*, MVZ, MSc. ${ }_{1,2}$, Juan Carlos GiraldoVillegas, MVZ, MSc. ${ }_{1,2}$

Departamento de Salud Animal, Universidad de Caldas, Manizales, Colombia

${ }_{2}$ Centro de Especialistas Mascotas, Manizales, Colombia

Recibido: 10 de abril del 2014

Aprobado: 28 de mayo del 2014

*Autor de correspondencia: Ricardo Andrés Correa-Salgado. Universidad de Caldas, calle 65 n. ${ }^{\circ}$ 26-10, Manizales, Colombia. Teléfono: (57) 6 8781500. Correo electrónico: ricardo.correa@ucaldas.edu.co

Cómo citar este artículo: Correa-Salgado RA, Giraldo-Villegas JC. Osteopatía hipertrófica secundaria a metástasis pulmonar de carcinoma mamario. Spei Domus. 2015;11(22):63-68. doi: http://dx.doi.org/10.16925/sp.v11i22.1157

Resumen. Propósitos: este artículo pretende reportar los hallazgos de un caso clínico de osteopatía hipertrófica. Tema: la osteopatía hipertrófica es un raro desorden paraneoplásico, asociado con el sobrecrecimiento doloroso del periostio de los huesos largos, normalmente desencadenado por neoplasias primarias o metastásicas de pulmón. Desarrollo: se presenta el caso de un Rottweiler, de 12 años, con osteopatía hipertrófica asociada con metástasis pulmonar de un carcinoma mamario. Conclusiones: se comentan los hallazgos clínicos, radiográficos y las posibilidades terapéuticas.

Palabras clave: canino, neoplasia pulmonar, osteofitosis periostales. 


\section{Hypertrophic Osteopathy Secondary to Pulmonary Metastases from Breast Carcinoma}

Abstract. Purpose: this article aims to report the findings of a clinical case of hypertrophic osteopathy. Topic: hypertrophic osteopathy is a rare paraneoplastic disorder associated with painful overgrowth of the periosteum of long bones, usually triggered by primary or metastatic lung tumors. Development: the case of a 12-year-old Rottweiler with hypertrophic osteopathy associated with pulmonary metastases from breast carcinoma is presented. Conclusions: clinical and radiographic findings and therapeutic possibilities are discussed.

Keywords: canine, lung tumor, periosteal osteophytosis.

\section{Osteopatia hipertrófica secundária à metástase pulmonar de carcinoma mamário}

Resumo. Propósitos: este artigo pretende relatar as constatações de um caso clínico de osteopatia hipertrófica. Tema: a osteopatia hipertrófica é uma rara desordem paraneoplásica, associada com o supercrescimento doloroso do periósteo dos ossos longos, normalmente desencadeada por neoplasias primárias ou metástases de pulmão. Desenvolvimento: apresenta-se o caso de um Rottweiler, de 12 anos, com osteopatia hipertrófica associada com metástase pulmonar de um carcinoma mamário. Conclusões: comentam-se as constatações clínicas, radiográficas e as possibilidades terapêuticas.

Palavras-chave: canino, neoplasia pulmonar, osteofitose periostais. 


\section{Introducción}

La osteopatía pulmonar hipertrófica (OPH), conocida en humanos como osteoartropatía hipertrófica, es un trastorno proliferativo perióstico difuso de los huesos largos [1], que en los humanos puede ser primario (paquidermoperiostosis), en el cual no se detecta una patología torácica o abdominal concomitante y tiene un factor de herencia autosómico recesivo [2], o puede ser secundario a procesos infecciosos o neoplásicos en pulmón; esta forma secundaria se presenta en humanos, perros y con menos frecuencia en gatos [3].

Aunque no se conocen los mecanismos patogénicos exactos, se sabe que se produce una excesiva vascularización perióstica periférica [4], la cual conduce a una congestión venosa que, a pesar de ofrecer un aumento en el aporte local de sangre, se encuentra pobremente oxigenada debido a la formación local de shunts arteriovenosos. Esto predispone a la hipoxia del tejido y estimula la proliferación de tejidos conectivos, lo que lleva a neoformacion ósea perióstica en falanges y huesos distales [5].

Existen tres teorías sobre su patogenia. La teoría neurogénica expone que la lesión torácica produce una irritación del nervio vago, que es la causante de la hipervascularización; esto se sustenta en que la vagotomía y el tratamiento con atropina suele disminuir las lesiones óseas [1]; también se ha involucrado a los nervios intercostales [6]. La teoría humoral propone como desencadenante un incremento de sustancias como prostaglandinas, ferritina, bradiquinina, estrógenos y hormona del crecimiento [7]. La teoría plaquetaria supone que la lesión pulmonar provoca una fragmentación plaquetaria que libera partículas a la circulación venosa; dichas partículas provocan una activación endotelial en la porción distal de las extremidades, liberando factores de crecimiento plaquetario que provocan una proliferación de tejido conectivo y periostio [8]. Esta teoría se apoya en que gran cantidad de pacientes humanos con opH tienen elevación en los niveles de factor Von Wilebrand [9].

La lesión más comúnmente asociada a la $\mathrm{OPH}$ es el tumor pulmonar primario de origen bronquial, pero también se ha reportado con metástasis pulmonares, neumonía, tuberculosis, cardiopatías congénitas, tumores esofágicos, dirofilariasis, spirocercosis, hepatozoonosis, e incluso, tumores abdominales como carcinoma hepático, vesical y prostático $[1,5]$.

La sintomatología de la OPH se limita a un dolor progresivo en los huesos largos, claudicaciones y rigidez, que por lo regular son signos más incapacitantes que los propios de la patología desencadenante [5].

Las radiografías de los miembros revelan la proliferación perióstica, principalmente en falanges y en metacarpos/metatarsos, y algunas veces logra ascender hasta húmero y fémur. Las radiografías torácicas generalmente evidenciarán la lesión desencadenante [3]. Sin embargo, no siempre es fácil detectar neoplasias pulmonares en radiografías, ya que solo se pueden detectar lesiones que superen los $5 \mathrm{~mm}$, por lo que es más factible detectarlos cuando la radiografía se toma en inspiración completa [10].

Si las neoplasias se encuentran en espacio subpleural, área hiliar o lobos pulmonares caudales, es probable que solo se detecten radiográficamente cuando superen los $10 \mathrm{~mm}$; para aumentar la posibilidad de detectarlos, se aconseja tomar cuatro vistas radiográficas [10]. La mayoría de perros con tumores pulmonares son asintomáticos hasta que estos superan los $30 \mathrm{~mm}$ [10].

El tratamiento curativo para la $\mathrm{OPH}$ consiste en la extracción de la masa torácica cuando esto es posible. Si dicha lesión es irresecable, una vagotomía intratorácica ipsilateral puede bloquear el reflejo neurovascular que se sospecha que está involucrado [11]. Ambas intervenciones muestran una mejoría evidente en 2 a 4 semanas [6].

En los casos donde la intervención quirúrgica no se pueda realizar, el uso de antiinflamatorios no esteroidales o corticoides puede aliviar la sintomatología durante un tiempo, aunque los estudios retrospectivos muestran que la mejoría es muy sutil [11] También se tienen datos anecdóticos de resultados alentadores con medicamentos inhibidores de los osteoclastos como los bifosfonatos [5]. Sin embargo, en cualquiera de los casos, la OPH es de un pronóstico reservado, debido a su relación principalmente con patologías de carácter maligno.

\section{Caso clínico}

Se presenta a consulta al Centro de Especialistas Mascotas de Manizales un canino hembra Rottweiler, de 12 años, por claudicación intermitente de los cuatro miembros, postración y anorexia. A la perra se le había practicado una mastectomía bilateral total por tumores mamarios múltiples ocho meses atrás; el resultado del estudio histopatológico de tres 
muestras tomadas de diferentes glándulas mamarias reveló un carcinoma tubular.

Al examen clínico se encuentra una temperatura rectal de $39,9^{\circ} \mathrm{C}$, inflamación de la porción distal de los cuatro miembros (figura 1), con dolor manifiesto a la palpación. La auscultación pulmonar no revelaba cambios.

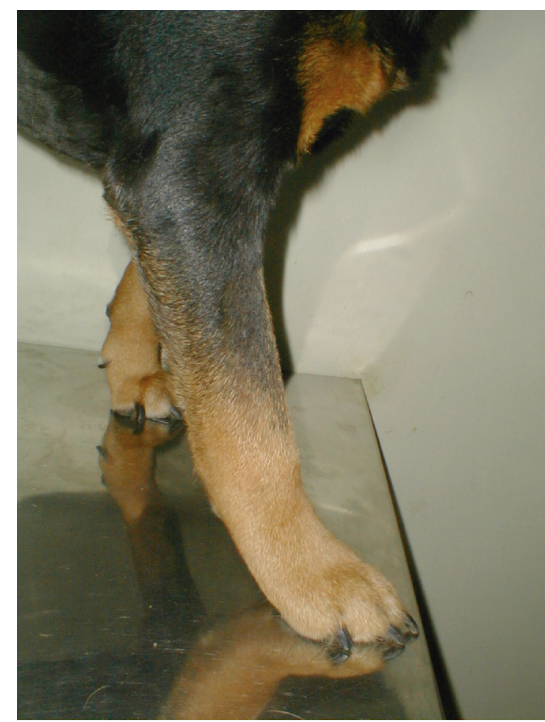

Figura 1. Paciente con inflamación de la porción inferior del miembro torácico

Fuente: elaboración propia

Se realizaron los exámenes señalados en la tabla 1.

Tabla 1. Exámenes realizados con su respectivo valor y referencia.

\begin{tabular}{|l|c|c|}
\hline \multicolumn{1}{|c|}{ Examen } & Valor & Referencia \\
\hline Hematocrito & $36 \%$ & $38-50 \%$ \\
\hline Hemoglobina & $12,2 \mathrm{~g} / \mathrm{dl}$ & $14,1-20 \mathrm{~g} / \mathrm{dl}$ \\
\hline Leucocitos & $17,8 \times 106 / \mathrm{ul}$ & $6-16 \times 106 / \mathrm{ul}$ \\
\hline Neutrofilos & $15,6 \times 106 / \mathrm{ul}$ & $3,23-10,85 \times 106 / \mathrm{ul}$ \\
\hline Linfocitos & $1,06 \times 106 / \mathrm{ul}$ & $0,53-3,44 \times 106 / \mathrm{ul}$ \\
\hline Bandas & $1,06 \times 106 / \mathrm{ul}$ & $0-1 \times 106 / \mathrm{ul}$ \\
\hline Plaquetas & $520 \times 103 / \mathrm{ul}$ & $170-510 \times 103 / \mathrm{ul}$ \\
\hline Creatinina sérica & $0,87 \mathrm{mg} / \mathrm{dl}$ & $0,5-1,5 \mathrm{mg} / \mathrm{dl}$ \\
\hline
\end{tabular}

Fuente: elaboración propia
La radiografía anteroposterior del miembro torácico izquierdo revela la proliferación de neo-formaciones óseas periósticas que comprometen la diáfisis del radio, la ulna y los huesos metacarpianos (figura 2).

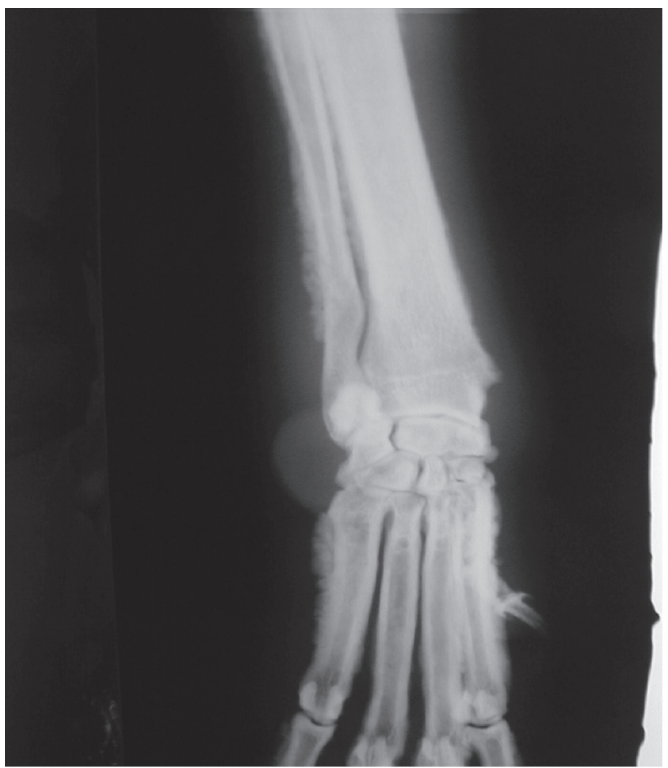

Figura 2. Radiografía anteroposterior de miembro torácico izquierdo que revela importante proliferación perióstica (flechas)

Fuente: elaboración propia

Con estos resultados, se sospechó de una OPH y se tomó una radiografía de tórax que reveló tres lesiones neoplásicas en los campos pulmonares caudales (figura 3), que en los estudios histopatológicos posteriores revelaron ser metástasis del carcinoma mamario.

Es importante anotar que a la paciente, ocho meses atrás, previo a la mastectomía, se le había practicado un estudio radiográfico de tórax en el que no se evidenciaron alteraciones.

La paciente se medicó con carprofeno (Pfizer, Nueva York, EE.UU.) 2,2 mg/kg/12h y se mantuvo con una sintomatología mínima durante tres semanas, evaluada diariamente por los propietarios mediante la encuesta de calidad de vida para pacientes con cáncer [12]. Luego de este tiempo reapareció la inflamación y la claudicación, momento en el cual se le practicó la eutanasia. 


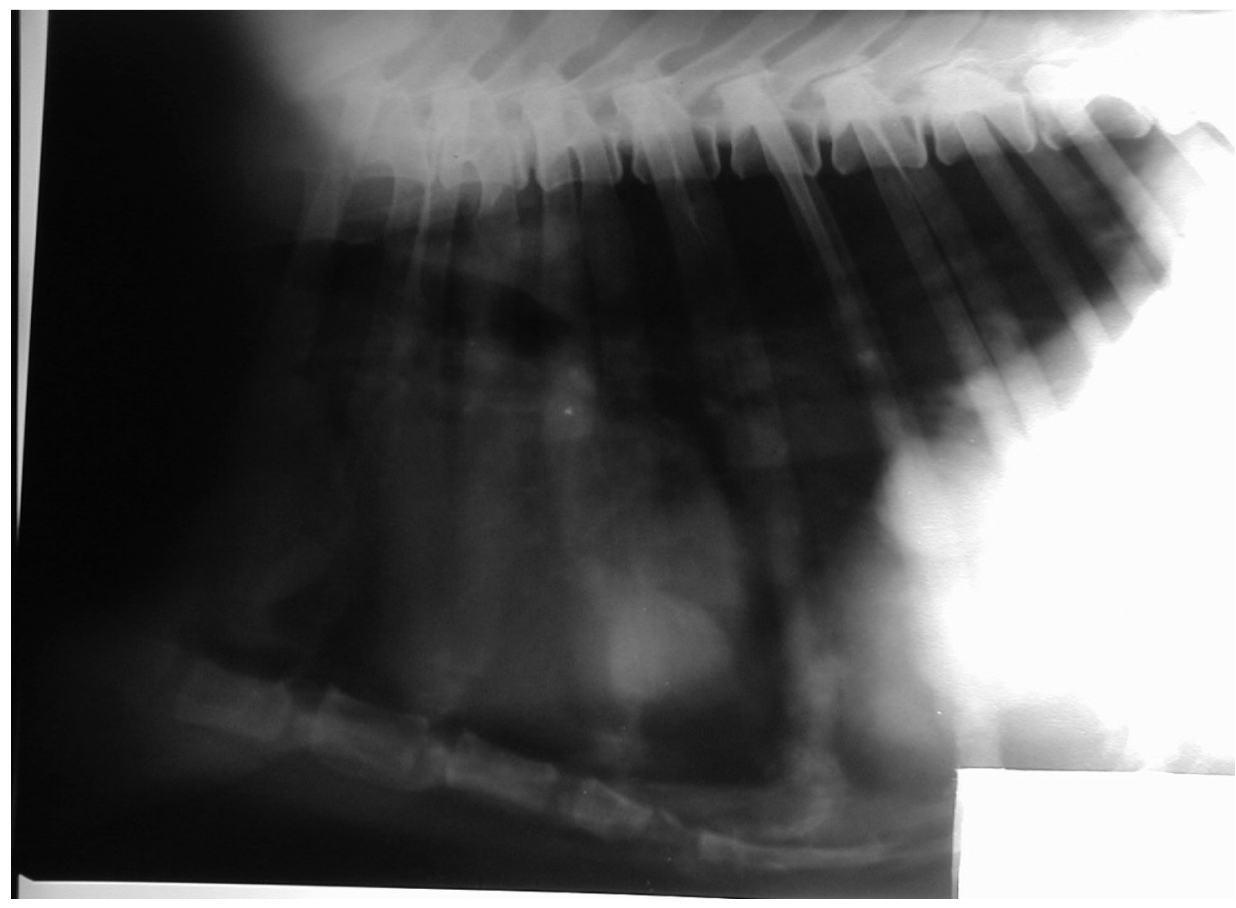

Figura 3. Radiografía laterolateral de tórax que muestra tres nódulos neoplásicos en pulmón Fuente: elaboración propia

\section{Conclusiones}

La OPH puede ser primaria o secundaria; la forma primaria hereditaria solo se reporta en humanos, mientras la forma secundaria es considerada un síndrome paraneoplásico que, aunque tiene escasa frecuencia en la práctica diaria, es una entidad a tomar en cuenta dentro de los diagnósticos diferenciales en pacientes geriátricos que son presentados a consulta con inflamación de la porción distal de las extremidades, máxime si dicha alteración está presente en más de una extremidad.

Es importante recordar que las lesiones tumorales pulmonares solo son apreciables en las radiografías cuando superan un tamaño de $5 \mathrm{~mm}$; por tal motivo, se debe informar a los propietarios que el hecho de no observarlas en las radiografías no implica que no existan. Esto pudo haber sucedido en el caso de esta paciente, pues no se observaron las neoplasias pulmonares en el momento de la mastectomía y muy probablemente ya existían.

Como reportan la mayoría de autores, en esta paciente la sintomatología se limitaba al síndrome paraneoplásico de osteopatía hipertrófica, con decaimiento, fiebre, anorexia y claudicación importante. En ningún momento, el propietario reportó signos respiratorios que sugirieran que la lesión primaria se encontraba en pulmón; de igual manera, el examen clínico y la auscultación no mostraba alteraciones del tracto respiratorio.

La literatura reporta múltiples opciones de tratamiento para humanos con esta enfermedad, pero la mayoría van encaminadas a paliar las lesiones neoplásicas pulmonares. En la literatura veterinaria, los reportes encontrados muestran que la mayoría de pacientes son eutanasiados, quizá por los malos pronósticos de las patologías desencadenantes.

Aunque la osteopatía hipertrófica es una patología de escasa presentación en la práctica clínica diaria, se debe incluir en los diagnósticos diferenciales de perros y gatos con claudicación e inflamación de la porción distal de los miembros. Adicionalmente, la radiografía debe considerarse un examen rutinario en pacientes con esta sintomatología, en la que los hallazgos suelen ser muy específicos para esta enfermedad. Una vez hallados los signos radiográficos en las extremidades, se debe hacer un análisis exhaustivo de la cavidad torácica y abdominal para encontrar la patología desencadenante del síndrome paraneoplásico; se debe advertir al propietario del 
paciente que dicho trastorno suele ser de un pronóstico muy desfavorable.

\section{Referencias}

[1] Yang WC, Lin SC, Liu TC, et al. Clubbed fingers and hypertrophic osteoarthropathy in a patient with squamous cell carcinoma of the lung. Kaohsiung J Med Sci. 2003;19(4):183-7.

[2] Matucci-Cerinic M, Lotti T, Calvieri S, et al. The spectrum of dermatological symptoms of pachydermoperiostosis (primary hypertrophic ostoarthropathy): a genetic, cytogenetic and ultrastructural study. Clin Exp Rheumatol. 1992;10(Suppl. 7):45-8.

[3] SánchezMA. Traumatología y ortopedia de pequeños animales. Madrid: McGraw-Hill; 1997.

[4] Roush JK. Doenças que afetam o osso em desenvolvimento. En: Birchard SJ, Sherding RG. Manual Saunders-Clínica de pequenos animais. São Paulo: Roca; 1998. p. 1197-1205.

[5] Liptak JM, Monnet E, Dernell WS, et al. Pulmonary metastatectomy in the management of four dogs with hypertrophic osteopathy. Vet Comp Oncol. 2004;2:1-12.
[6] Fox L. Trastornos paraneoplásicos. En: Bonagura J, Kirk R. Terapéutica de pequeños animales XII. México D. F.: McGraw-Hill; 1997.

[7] Uchisako H, Suga K, Tanaka N, et al. Bone scintigraphy in growth hormone-secreting pulmonary cancer and hypertrophic osteoarthropathy. J Nuclear Med. 1995;36:822-5.

[8] Wegrowski Y, Gillery P, Serpier H. Alteration of matrix macromolecule synthesis by fibroblasts from a patient with pachydermoperiostosis. J Invest Dermatol. 1996;106:70-4.

[9] Matucci-Cerinic M, Martínez-Lavin M, Rojo F, et al. Von Willebrand factor antigen in hypertrophic osteoarthropathy. J Rheumatol. 1992;19:765-9.

[10] Baez JL, Sorenmo KV. Pulmonary and bronchial neoplasia. En: Textbook of respiratory disease in dogs and cats. Filadelfia: Saunders; 2004.

[11] Da Silva AF, García E, Schoff F, et al. Osteoartropatia hipertrófica secundária a neoplasia pulmonar: Relato de caso. Rev Port Pneumol. 2009;15(4):705-11.

[12] Yazbeck KVD, Fantoni DT. Validity of a health-related quality of life scale for dogs with pain secondary to cancer. JAVMA. 2005;226(8):1354-8. 